Euskal ikerketen aldizkaria | Revue d'études basques |

Revista de estudios vascos | Basque studies review

$4 \mid 1999$

Numéro IV

\title{
Notes d'ethnolinguistique : l'oiseau et la pluie en Soule et Basse-Navarre
}

\section{Charles Videgain}

\section{OpenEdition}

\section{Journals}

Édition électronique

URL : http://journals.openedition.org/lapurdum/1564

DOI : 10.4000/lapurdum.1564

ISSN : 1965-0655

\section{Éditeur}

IKER

Édition imprimée

Date de publication : 1 octobre 1999

Pagination : 223-245

ISBN : 2-84127-156-0

ISSN : 1273-3830

Référence électronique

Charles Videgain, « Notes d'ethnolinguistique : l'oiseau et la pluie en Soule et Basse-Navarre », Lapurdum [En ligne], 4 | 1999, mis en ligne le 01 juin 2010, consulté le 29 janvier 2020. URL : http:// journals.openedition.org/lapurdum/1564; DOI : 10.4000/lapurdum.1564 


\title{
X. VIDEGAIN
}

\section{NOTES D'ETHNOLINGUISTIQUE : L'OISEAU ET LA PLUIE EN SOULE ET BASSE-NAVARRE}

\author{
UMR 5478 CNRS, UPPA \\ Juxue : Erten da : "To! okhiloa ai duk \\ arbolari kokha, okhilia egarri, euri inen dik!". \\ Okhiloak etziela nahi putziain xahatzen ari eta \\ gio okhiloa egarri zela eta edaten zien gisala \\ ostoetarik erori ura, euria ari zelaik bakharrik \\ eta koskaka ari delaik arbolari : "To egarri. \\ duk! Euri nahi fite!". Okhiloak zangoak biziki \\ garbi ditu : etzielakotz nahi putziain xahatzen \\ ari. Eta gio ahatik egarri ederrik pasatzen. \\ Erten, erten. "Eztuk nahi zikhindu, egarriaki- \\ lan egoin hiz !".
}

On le dit: "Tiens le pivert est en train de cogner dans l'arbre, le pivert a soif. il va pleuvoir". Que le pivert n'avait pas voulu nettoyer la mare et puis que le pivert était assoiffé et qu'il buvait seulement, paraît-il, l'eau tombée des feuilles, et quand il cogne l'arbre: "Tiens! Il a soif! Il veut pleuvoir!". Le pivert a les pattes très propres. C'est qu'il ne voulait pas nettoyer la mare. Et ensuite cependant il passe par de belles soifs. On le dit, on le dit. "Tu n'as pas voulu te salir, tu resteras assoiffe" !

Le présent travail veut contribuer à ce qui pourrait être un bestiaire en domaine basque basé sur la tradition orale, et ce essentiellement à partir de données de Soule et Basse-Navarre : il veut aussi renseigner sur les variations qui imprègnent les données recueillies sur le terrain et qui débordent largement le niveau lexical ou purement linguistique auquel s'attache généralement le dialectologue.

Je me limite ici aux informations recueillies en cours d'enquête de thèse (Videgain 1989) ou dans le cadre des relevés sur le terrain pratiqués pour le compte de l'Atlas Linguistique du Pays Basque (EHHA)' à partir de la question 'pivert'. Mais les informations recueillies m'ont poussé à réunir des observations obtenues à l'égard d'autres oiseaux que le pivert et qui méritent le rapprochement.

En effet si la question 'pivert' laisse apparaître une carte monolexématique ou presque, on verra que la variation ne manque pas à d'autres niveaux que le niveau lexical, en particulier au niveau phraséologique, et dans les récits qui ont été recueillis concernant une relation faite entre un oiseau et la pluie. 
J'ai tenté d'ordonner l'ensemble de ces observations d'ordre lexicologique et ethnolinguistique selon le schéma donné ci-dessous. Le lecteur jugera ensuite à la lecture d'une bonne part des observations enregistrées au magnétophone auprès de divers locuteurs si je n'ai point trop sollicité les témoignages pour les ordonner selon le plan suivant :

1. Le signifiant basque pour 'pivert' offre des variantes d'un même vocable : okhilo en Basse-Navarre, ükhül, caractéristique de la Soule. À noter la forme propre à Bardos : oskil. Le Labourd présente aussi okil, forme attestée en Biscaye, et dont la forme à palatale okill couvre plus que le Guipuscoa. Des zones plus réduites en Navarre ont une consonne à l'initiale : kokill à Xuarbe, Alcoz... et tokilo en Baztan et à Eugi, Cilbeti, Mezquiriz... (voir le détail de l'aréologie dans la carte 05260 de EHHA-1, à paraître).

1.1. Des formes composées sont possibles : ükhülberde, ükhülbeltz, borthüko-ïkhül.

1.2. Deux vocables différents, okhilo et koskotari, plus une forme motivée euri-eskale peuvent coexister.

1.1. et 1.2. sont imputables à une différenciation des référents, les locuteurs connaissant ou non et désignant ou non de façon distincte diverses variétés d'oiseaux parmi les Picidae.

2. La métaphore basée sur la désignation du pivert est très courante en Soule et Basse-Navarre, elle paraît moins spontanéc ou courante vers l'Ouest. La justification de la connotation négative de la métaphore offre aussi une variation. Certains locuteurs considèrent en effet qu: le comportement du pivert signale de l'habileté en dépit d'un emploi phraséologique sévère pour le pivert.

3. Presque tous les locuteurs établissent un lien entre le comportement d'un oiseau et la pluie prochaine.

3.1. Ce lien est exprimé dans le discours sous diverses formes.

3.1.1. Sous forme libre tel qu'un énoncé empirique d'observation météorologique.

3.1.2. Sous forme codée tel un proverbe.

3.2. Le discours varie sur le contenu par rapport à la pluie.

3.2.1. L'oiseau annonce la pluie : c'est l'énorme majorité des cas.

3.2.2. L'oiseau annonce la sécheresse (ici et là et en Labourd côtier près de la Nivelle).

4. En ce qui concerne le protagonisme de l'oiseau annonciateur de pluie la variation marque aussi le protagoniste (cf Propp 1965).

4.1. L'oiseau annonciateur est le pivert, majoritairement.

4.2. Il peut être le milan, ülhaintxa. 
4.3. Il peut être le 'milan blanc' minuzuri ou mülüxuri; ou bien la 'buse', buzoka, ou bien aphobelatxa, le 'busard' sans doute, ou la 'pie' phika, ou la 'corneille' belatxa, mais aussi d'autres animaux, tous rapaces ou corvidés, sans parler de behien bideko emazte xuria, littéralement " la dame blanche du chemin des vaches' pour le 'pernocptère'.

5. La soif prêtée à l'oiseau peut être fondée à partir d'un récit étiologique ou récit mythique.

5.1. En Soule, il n'a pas été recueilli de narration dont le pivert soit le héros

5.2. En Basse-Navarre, il n'a pas été recueilli de narration dont le pivert ne soit le héros.

6. La raison du châtiment dont l'oiseau protagoniste est victime offre aussi matière à variation.

6.1. Le châtiment est la conséquence d'un méfait inconnu.

6.2. Le châtiment est la conséquence du manque de solidarité de l'oiseau (axe horizontal).

6.3. Le châtiment est la conséquence d'une insoumission à une force supérieure (axe vertical).

\section{Illustrations et commentaires de cette schématisation}

Je donne ici en les ordonnant selon le plan ci-dessus les commentaires que les locuteurs ont formulés. Le texte basque est suivi d'une traduction. Pour le type de transcription, on se reportera aux conventions indiquées dans Videgain 1989, et qui s'inspirent des travaux sur l'oralité dont ceux de $\mathrm{Cl}$. Blanche-Benveniste 1987.

Je commence par rassembler les informations de type taxonomique sur le pivert et oiseaux de la même famille. Les témoins distinguent divers oiseaux ou bien caractérisent l'aspect de l'oiseau désigné.

Mondragon: Oneik daus lau-bos klase. Au okilla. Da au pentzetan dot dala aunditxuenetarikoa... burúgorrixa. Oneik askó jaten dabe txiñddúrrixa.

Là aussi il y a quatre ou cinq variétés. Je crois que celui-ci (c'est-à-dire l'oiseau montré dans l'iconographie de l'enquête, Picus viridis) est un des plus grands, à tête rouge. Celui-ci mange énormément de fourmis.

Valcarlos : Orí, euri eskalea zerbeit érten órri... mii luzé bat badu arék... arek zinurrriaǩ biltzén tu miiarekin... ori yateko iltzeakin ta errótik atera miĺa... ala erten dúte.

Celui-là, on l'appelle quelque chose comme le quêteur de pluie, celui-là a une longue langue, celui-là ramasse les fourmis avec sa langue, si on veut le manger, quand on le tue il faut lui arracher complètement la langue, on le dit ainsi.

Esterençuby : Koxkotaria gaztain koloria da komunzki, gris-aire. Gio bada handia, bada okhiloa, haundio hua, hua re koxkotaria, hura beno geiho re, eta hartaik bizi da, aldiz koxkotariak arthoa re jaten du... (Denbora kanbio delarik) ordian iten du horrek kuku ku kukuku, eurieskalia deitzen dute hua, okhiloa eta 
eurieskalia bat dira, hori arbole batetik besterat joaiten da, kukukukuku inez, euri eskale bo euria heldu duk, euri re doala, eurieskalia mintzouk, euria heldu duk; gio bada beste klasa bat hoietan, bada ere deitzen dutena okhilo beltza, gainian da hua eta beste kantu klasa iten du harek hü, badu delako eurieskale horrek e mateletan gorria, hak e badu oiloak bezala, kukukusta bezalakoa du okhilobeltzak.

Celui appelé koxkotari, il est communément de couleur grisâtre. Ensuite il en est un plus grand, celui appelé okhilo, il est plus grand celui-là, lui aussi il est koxkotari (= il cogne dans les arbres), mais plus grand, et il vit de cela (de creuser des trous), alors que le koxkotari par contre il mange aussi le mais. Quand le temps change, alors il fait kuku ku kukuku, celui-là on l'appelle le quêteur de pluie, okhilo et le quêteur de pluie ne font qu'un, il va d'un arbre à l'autre, en faisant kukukukuku, ah voilà la quêteur de pluie, la pluie arrive, que la pluie va venir, tiens le quêteur de pluie chante! il va pleuvoir. Ensuite il existe une autre sorte parmi eux, il existe aussi celui qu'on appelle okhilo noir, il l'a la partie noire) sur le dessus, et celui-là produit une autre sorte de chant hü, et le dénommé quêteur de pluie a lui aussi du rouge sur les joues, mais le okhilo noir, comme la poule, a une crête.

Okhilo beltz est certainement le 'pic noir', Dryocopus martius (Peterson, 1984, 254-255).

Larrau : A lekukoa : Bi kusk emaiten dü eta bestaldilat badua zalhe deus $e$ higitzen den. Aogaitza, biek eztie ber mintzoa, elgarren ahaidek ere mintzoan, horrek krukrukrukru egiten.

- B lekukoa : Bena ükhülberdiak markhatzen dü ebia, nabetan sartzen dük ebia ai denian.

Témoin A : Il donne deux coups sur le tronc et il va voir très vite de l'autre côté voir si quoi que ce soit bouge. Pour le mauvais temps, aussi, les deux ont le même chant, ils sont apparentés aussi par la voix, celui-ci fait krukiukru.

Témoin B : Mais celui appelé ükhülbeltz ('pic noir') il annonce la pluie, il entre dans la vallée quand la pluie est là.

L'oiseau appelé ükhülbeltz a été répertorié comme le 'pic noir', Dryocopus martius (Peterson, 1984, 254-255). Pour le comportement autour du tronc, voir plus loin.

Larrau : (Bohortxoia) ehaiten denian, mihia behar da elkhi arrunt, ünhürrian bizi beita, eta ükhüla ber gaiza, süstut borthü-ïkhüla.

Quand on tue l'étourneau, il faut lui arracher la langue aussitôt, parce qu'il vit de fourmis, et pour le pivert c'est la même chose, surtout le pivert de montagne.

Borthü-ükkhüla où borthü fait référence à la zone d'estive en montagne, semble être une autre variété mais nul renseignement supplémentaire n'a été demandé.

Sainte-Engrâce : Ükhüla, beltza, ñabarra, eta berdia eta beste txipi bat beste süerte bat düzü, papho gorri bateki.

Le pivert, le noir, le fauve et le vert, et puis un autre qui est tout petit et qui est une autre variété, avec un cou rouge. 
Je donne maintenant les informations de type encyclopédique. On notera la référence faite aux trous creusés dans le bois, action considérée comme caractéristique du pivert et dont la désignation onomatopéique koxkotari rend compte, mais aussi la référence au goût de fourmi prêté au pivert en raison de son alimentation. La propension qu'aurait le pivert à entamer le bois peu sain est relevée par plusieurs locuteurs. Enfin, l'abeille est aussi mise en relation par plusieurs locuteurs avec le trou creusé par le pivert et qui sert d'abri à l'essaim.

Lejoa : Okile bai. Ori da suloetan abie iten dauena. Onek múskerrak moduen kantetan dau. Muskerrak bere $k u-k u-k u-k u$. Onek bere goixetan.

Le pivert. C'est celui qui fait son nid dans les trous (d'arbre). Celui-ci chante à la façon du lézard vert. Le lézard vert aussi fait aussi ku-ku-ku-ku. Et celuici aussi le matin.

Renvoi est donc fait au lézard vert dont plusieurs locuteurs témoignent qu'on peut l'entendre chanter (cf. EHHA-1, question 04070 'lézard vert').

Ezkio-Itsaso : Okillek úmek etátze'ittú gaztiñan zulóa indá, zulóa beák éî̀ eta jenéralen okillek zúlatzen dó árboleá ustéldutakoá..., erdíko muñén eo..., árbola sánoa ez, barrún uékua daukén árboleá zúlatzen do mókokiñ... ta án etátze ittú umék... Eztie gózok játekó, txingórrie játen dué ta.

Le pivert fait ses petits dans le châtaignier après y avoir creusé un trou, un trou qu'il fait lui-mème, en général il perce dans un arbre pourri, vers la moelle au milieu ou bien..., un arbre sain, non, au milieu il creuse de son bec l'arbre qui a déjà un creux, et là il élève ses petits, les piverts ne sont pas agréables à manger, c'est qu'ils mangent des fourmis.

Hernani : Txingurri gustu izate'u ikáragarriya, txingurriya jate' baitu orrek...

Il a un goût terrible de fourmi, parce qu'il mange les fourmis.

Fontarabie : Beste klase bat da, okillak txingúrriak jaten'ittu... Okikiki kantatzen du, orain etorriko die.

C'est une autre sorte, le pivert mange les fourmis, il chante en faisant okikiki, ils vont arriver maintenant.

Saint-Jean-de-Luz : Añitz aldi orrek intako (egindako?) zilloan erlia sartzen da...

Très souvent dans le trou qu'il a fait entre l'abeille.

Bardos : Oihantzia barnian du, arbolaan barnian du, behin arbola bateri buruz, arbola xahar bateri buruz, joka ari da, eta itzulia ingurian johan da ikhustea xinaurriak eztirenetz bestalderdian jali, bere bizimoldia da, xinaurriak eta mihia latza du eta mihian sartzen tu eta iresten. Pherde eta gorri bada, bürügorri eta beste bat ttipixo, "pic épeiche", de la famille des grimpeurs, buztana biziki azkarra ute.

Il a son nid à l'intérieur, il l'a à l'intérieur de l'arbre, d'abord il s'attaque à frapper un arbre, à un vieil arbre, et ensuite il va faire le tour pour voir s'il n'y a pas de fourmi qui sorte de l'autre côté, c'est sa façon de vivre les fourmis, et il a la langue rugueuse et il les met sur sa langue et les avale. Il y a le vert et le 
rouge, une tête rouge, et un autre, plus petit, le "pic épeiche, de la famille des grimpeurs", il ont une queue très solide.

Armendaritz: Jenealian, arbole hunkian ba da ari, arbole zaharretan, arbole freskoa etzi ez hola zilhatzen, harrak eta zinurriak gehienik omen jaten tzi, oai zonbeit jendek jaten tzie leheno entzun tzit janik eta izigarriko zinurri gustia omen du...

En général, il travaille dans l'arbre déjà atteint, dans les vieux arbres, il ne creuse pas tellement l'arbre qui est frais (en bon état), il mange dit-on surtout des vers et des fourmis, maintenant, il y a des gens qui le mangent, autrefois j'ai entendu dire que si on le mange il a dit-on un goût de fourmi...

Lantabat : Okiloziloa... erliak ee frangotan erleumiak yoaiten zielaik kafiatik, airatzen zielaik, eta okiloziluetan sartzen...

Le trou de pivert, très souvent les abeilles, et quand les essaims quittent leur nid, quand ils s'envolent, très souvent ils rentrent dans un trou de pivert.

Arbouet-Sussaute : Xinhurriak jaten bitu, eztüzü ihize huna...

Comme il mange les fourmis, ce n'est pas un gibier agréable (à manger).

Larveveau : Khur khur khur khur moskoaekin eta bestalderdirat joan zilhatia denetz arrunt, eta adar zilhoetan egoiten.

Il fait khur khur khur avec son bec et il file aussitôt de l'autre côté (de la branche) et il reste dans les trous de branche.

Larceveau : Ez, ez, okhiloa jateko erten dizie eztela hun, izigarri xinhurri gustia badiela, nik eztiakizut.

On dit que le pivert n'a pas bon goût si on le mange, qu'il a un goût terrible de fourmi, moi, dites, je ne sais pas.

Pagolle : Haitz zaharretan egiten diela habia eta gio haren habietan erliak e, erlekhümiak egiten titzie.

On dit qu'il fait son nid dans les vieux chênes, et ensuite dans leurs nids, les abeille's, elles y ont leurs essaims.

Saint-Etienne de Baïgorry : Hau dea üñhürriz bizi dena? Mihia elkhitzen dü khanpoat et'oro ünhürriz bethetzen beita ta hak gaintitzen oro, hartako jateko ezta gozo.

Est-ce celui-ci qui vit de fourmi? Il tire la langue au dehors et comme il la remplit toute de fourmis il les avale toutes, c'est pour cela qu'il n'a pas bon goût pour être mangé.

Domezain : Ah! ez, okhiloak eztu hola egiten, okhiloa harren xerkatzen ari da arbolian, tak tak jo eta bestaldetik so elkhitzen denetz. Ezta astoa, batee astoa, erten dute ba, okhiloa! eztakit zendako erten duten okhiloa bena astoa, ez apeentziaik e, gilletik so egiten diena ezta astoa eh! ezta har bat elkhiko alderdi huntaik, hantik elkhiko da, giiletik.

Ah le pivert ne fait pas ainsi, le pivert est en train de chercher des vers dans l'arbre, il frappe tac tac et va voir de l'autre côté pour voir s'il en sort. Ce n'est pas un imbécile, pas du tout un imbécile, on dit oui pivert! Je ne sais pas pour- 
quoi on dit pivert mais il n'est pas bête du tout, loin de là, il va voir par derrière, il n'est pas bête hein! Il ne va pas sortir de ver de ce côté-ci hein! Il en sortira par là derrière (oủ l'on voit que le comportement prêté à l'oiseau relève ici d'une logique favorable au pivert).

Alçay : Erran bat da, zühainian berdea, beltza zur idorretan berdin, ürzaka ai ginelaik, ürzoik ezpazen, frankotan e thiatü. Erraiten düte mihia behar zaola thiatü, phüntü batez zernahi jaten beitzen eta mihia behar zaola elkhi, bestelan ünhürri rıstua badiela, ünhürri hanitx juten beitie.

C'est un dicton, dans l'arbre c'est le vert, le noir par contre tout aussi bien dans le bois sec, quand on était à la chasse à la palombe s'il n'y avait pas beauoup de palombes on le tirait souvent aussi. On dit qu'il faut lui arracher la langue, à une époque comme on mangeait n'importe quoi, il faut donc lui tirer la langue, sinon il a un goût de fourmi, parce qu'ils mangent beaucoup de fourmis.

Sainte-Engrâce : Selon le témoin le pivert donne deux coups sur le bois et va voir de l'autre côté s'il a percé le bois.

Esquiule-Féas : Ükhülak hoi egiten dü bee bizigarriaen deitzeko, üñhürrik eta, üñhürria joiten den lekhila atheatzen da, bada bi ükhül süerte, ükhül berde eta bertzia, xui ta beltz bezala beita, ttipi, haikbadizü mihi bat lüze eta trrtrrtrrek eta ümen mihia hedatzen ümen dü eta üküküküküian nun eta üñhurrik atheatzen beitia eta hartan lothüik bezala dire.

Le pivert il fait ceci pour appeler sa nourriture, les fourmis et puis, la fourmi sort à l'endroit où le pivert frappe, il y a deux pics, le pic-vert et l'autre, qui est comme noir et blanc, un petit, celui-là a une longue langue et trrtrrtrrk et dit-on il étale sa langue dit-on et ükükükükü de telle sorte que les fourmis sortent et restent là comme collées.

Je réunis dans les lignes qui suivent les indications sur les métaphores ou comparaisons basées sur le pivert. Elles sont toujours à connotation négative et s'expliquent parfois de façon explicite par le comportement prêté au pivert et dont le chapitre précédent a pu donner une idée. On trouvera des remarques allant dans le même sens dans Rolland 1887. Celui-ci cite ainsi :

Le pic vert donne un coup de bec dans l'arbre et passe de l'autre côté dans l'arbre voir s'il est traversé (Finistère, Souvestre). À chaque coup de bec, les pics vont voir derrière s'ils n'ont pas traversé l'arbre (Saintonge, Jonain). Plus loin, Rolland donne la "vraie raison" de ce comportement :

Le pic, en réalité, va voir si le bruit de ses coups de bec n'a pas fait sortir de l'autre côté quelque insecte.

Certains de nos témoins prennent la défense du pivert et son comportement, signe de paresse ou sottise pour les uns, est une marque positive d'habileté pour d'autres.

Voici donc les remarques d'ordre phraséologique.

Uhart-Cize : Okiloa erten zen puxka at hóla zozóa zelaik noaiti... ze mutiko okílua.

Pivert on le dit à quelqu'un comme cela qui est un peu idiot (litt. 'merle'), quel garçon pivert (idiot)! 
Armendaritz: Ba, okhiloa, etzu habila, habila hua ere, etzu habila, eta egoiten da zangoekin hola goiti so, oai arrunt hola ezarriz bezala, hola, ta ari duk okhiloa bezala, erten tzu jender...

Oui, dites, le pivert, il n'est pas habile, celui-là non plus, il n'est pas habile, et il reste là sur ses jambes comme cela à regarder vers le haut, maintenant s'il est ainsi tout à fait installé, ainsi, il s'y fait comme le pivert, on le dit d'une personne...

Esterençuby : Tontoskoa delakotz, bere haat-hunatetan sobera untsa bere buria gobernatzen eztiena, erraiten da okhiloa!

Quand il est un peu bêta, quand il ne sait pas se gouverner lui-même dans ses allées et venues et démarches, on dit : pivert!

Gamarthe: Hola puska bat gaizo-aire delaik, "gaizoa, ze okhiloa iten dien horrek!" hala erten da, bo! Gaizo, puska bat.

Quand quelqu'un a un air plutôt demeuré, "le pauvre, quel pivert il fait" ! On le dit ainsi, bof! Un peu demeuré.

Garris : I : Ta jendetan zendako erten da, ze okiloa den? - L : Eztelakoan sobera abila, artako dakozie erien... - I : Lanian... - L : Ez, ez, amiñi at bee gisakoa balin bada, okiloa duk.. - I : Buruz, ez biziki erne... - L : Ez intelijenta..

$\mathrm{E}$ : Et pourquoi dit-on de quelqu'un : quel pivert? - T : Parce qu'il n'est pas trop habile, c'est pour cela qu'on l'emploie. - E : Au travail... - T: Non, non, un peu si c'est quelqu'un de particulier, c'est un pivert. - E : Pas très vif, de la corvelle... - T : Pas intelligent...

Lantabat : (Jendetan) Eztena abila ta eztelaik plaxenta ni(k) da(k)ita, okhiloa hutsa baita... ez adreta.

Quelqu'un qui n'est pas habile ou quand il n'est pas sympathique, est-ce que je sais? Parce que le pivert est creux, pas adroit

Larceveau : Deurrienokhiloa!

Pivert du diable (employé comme injure) !

Ordiarp : Eta bai ezpada tutafet normal ; ze ükhüla hizan! Nül dela.

Et oui, si quelqu'un n'est pas tout à fait normal, quel pivert tu fais! Il est nul.

Aussurucq : Ezpauzü gustüko jente bat erraiten da : ükhüla!

Si quelqu'un ne vous plaît pas, on le dit : pivert!

Larrau : Bo hüntza bezalaska, ber zentzüsia, eztela hardit batere, espiritüz mantsoska edo.

Oh! Un peu comme le hibou (employé métaphoriquement), c'est à peu près le même sens, qu'il n'est pas du tout vif, qu'il est très lent d'esprit ou bien...

Alçay : Astoxka denai, ükhül bat, edo ixilik aise egoiten eztenai.

$\dot{A}$ un âne, un pivert, ou bien à celui qui ne se tait pas facilement.

Chéraute : Fundantzia txipiko norbait, hanitx balio eztiena... Baduzü (= badoazü) ikhustea üñhüri jelkhi denetz, hartakotz baduzü, ez eta jakiteko xilho 
denetz ez. Eztüzü esprabi kholte baten jotia? Ünhürri behala elkhiko düzü barneti. Hartakotz. Eztüzü sobea ükhül.

Quelqu'un qui n'a guère de fonds, qui ne vaut pas grand chose. Il va voir si des fourmis sortent, c'est pour cela qu'il va voir, et non pas pour voir si le bois est percé. Vous n'avez jamais expérimenté de frapper sur un piquet? La fourmi sort aussitôt de dedans. C'est pour cela. Il n'est pas si pivert que cela en fait (l'action est donc interprétée comme préméditée et efficace).

Chéraute : Gizon pergüt, gizon ükhüla, ükhüla deitzen düzü un vaurien.

Un triste individu, un homme pivert, on appelle pivert celui qui est un vaurien.

Sainte-Engrâce : Ze ükhüla, erraiten üpho bat bezala...

Quel pivert, on le dit comme injure.

Esquiule-Féas : Jentetan ezta favorable ez ükhül, ez, ez, ez, bee gisako edo ez nahi konprenitü, ez sobea plomin, astua izan gabe bena berriz bütus bee ideien baakizü bekhoi, égoïste, ezpadü konprenitü, ezbadü behatü nahi besten erraner, bethi...

Oh si on le dit de quelqu'un ce c'est pas favorable que pivert, non, non, non, de celui qui est un peu spécial, ou qui ne veut pas comprendre, pas trop d'aplomb, pas vraiment un âne mais toujours buté dans ses idées, vous savez, égoîste, s'il ne veut pas comprendre, s'il ne veut pas écouter ce que disent les autres, toujours...

Esquiule-Féas : Gizon hil bat, ükhül bat, bü txipi bat.

Un homme fade, un pivert, une petite cervelle.

Après ces informations d'ordre taxonomique ou qui rendent compte d'un mouvement phraséologique, viennent ensuite les commentaires qui établissent un lien entre l'oiseau et la pluie. Ces observations de type météorologique sont souvent sous forme libre mais peuvent aussi prendre la forme codée d'un proverbe ou d'une sentence. Dans un premier temps, je donne seulement les textes qui ne sont pas des récits et dans lesquels le pivert est impliqué.

Errigoitia : Okille negárres dabil, egarri de esaten du guk kikikikiki iten dauenien, okillé egarri dé esaten du guk, euri eske egarri danien ur eske egoten da.

Le pivert est en train de pleurer, il a soif, nous le disons quand il fait kikikiki, nous disons le pivert a soif, il est en train de réclamer de l'eau.

Marquina-Etcheberria : Ki, kikikiki! (egiten duenean) okille egárri dalá, láster ekárriko dáu eurixe, e ?, okílle egárri dả ta... Eguáldixe kámbixue aukenin da... kikikiki ! da lastér da euríxe okille egárri dá ta, esáten da...

(Quand le pivert fait) Ki, kikikiki, on dit que le pivert a soif, il va vite apporter la pluie, parce que le pivert a soif, le temps va changer, kikikiki, la pluie sera. vite là, le pivert a soif, on le dit.

Ahetze : Euria egin bear duenian kokoko asten da arbolari... euri galdezka: ari denian.

Quand il va pleuvoir, il (le pivert) commence kokoko sur l'arbre, quand il se met à réclamer la pluie. 
Bardos: Hunek e, oihuka ari delaik, iten du oihu partikular bat, to uri beharra uk! eta erranak, erran xaharrak.

Celui-ci aussi produit un cri particulier, tiens il doit pleuvoir, c'est un dicton, de vieux dictons.

Saint-Etienne de Baïgorry : Okiloak ez du mihia ateratzen egarri delarik kanporat? Erran nahi baita fuil, füi, fuii, hola kurritzen da biziki seko delarik. Idorte handia delarik mihi holako luze bat duk; eta ihitzarekin edo airean bildu nahi dik humiitatia. - I : Punitia balitz bezala? - L : Punitia ez, idortea du señalatzen, lehengo xaharrer hala aditu deet.

Est-ce que le pivert ne sort pas la langue quand il a soif? C'est-à-dire fui fui fui, il déambule ainsi quand il fait très sec. Quand c'est la grande sécheresse, il a une langue longue comme cela; et avec la rosée ou dans l'air il veut recueillir l'humidité. E : Comme s'il était puni? T : Puni, non, il indique la sécheresse, je l'ai entendu ainsi aux anciens.

Gamarthe; Okhiloa mintzo delaik, euria markatzen du hak e, hala erraiten dute; okhiloa le pivert da..

Quand le pivert parle, il annonce la pluie, on le dit comme cela, okhiloa c'est "le pivert".

Béhorléguy: Gio erten dute okhiloa ari delaik, euria markhatzen diela kantuz ari balinbada, euri-eskalia dela,... kantutik ageri uxu. .

Et puis on dit que quand le pivert s'y fait, lui annonce la pluie, s'il est en train de chanter, ah dites cela se connaît à son chant.

Domezain : Ah idortia orroaz ari delaik eta üdan, hua entzüten delaik, to idortia inen dik, okhiloa ezagun duk, nik hala entzun diat, eztakit hala denetz, kax kax kax ari uk zurian eta erten die xindurria jaten diela eta kaxkatzen dik xindurriak khanpoat joan dizten, ah entzun diat ; lehen bazian geiho, phika e biziki güti dük.

Ah la sécheresse est là quand il se met à crier, et en été, quand on l'entend. tiens il va faire de la sécheresse, çà se reconnaît au pivert, moi je l'ai entendu ainsi, je ne sais s'il en est ainsi, il est en train de faire kax kax kax kax dans le bois et on dit qu'il mange les fourmis et il cogne afin que les fourmis s'échappent au dehors, ah je l'ai entendu, autrefois, il y en avait davantage, la pie aussi il y en a très peu.

Larrau : Bai ükhülak badizü bee zea, lehenokuek, gue aitzinekuek sinheste gaitza zizien, ezpeitzen ordin ez, (meteorik) eta kabalen zea zertzen züzün, jentiak bazizün bee hartü zea eta kabalian ganua, l'instinct, ganua, eta ükhüla, gük hizkar hortan gue etxeik ikhusten beitügu, oihan hegi hoi, ordian, oihan hegi hortun ai denin ükhüla, süstut petik hol'elkhitzen haietaa, denboa huna markhatzen dizü ; aldiz pli pli pli behea, nabetaat ehaisten denin ordin kuntraiua düzü, ükhüla behea pli pli pli hua ebia segür.

Oui le pivert a son truc, ceux d'autrefois, ceux d'avant nous avaient une croyance énorme, parce qu'il n'y avait pas alors de météo, et on prêtait attention au truc des bêtes, les gens prenaient en compte ce truc, l'instinct, l'instinct, et le pivert, nous sur cette petite crête nous le voyons depuis la maison, cette lisière de 
forêt, et quand le pivert s'y fait sur cette lisière de forêt, surtout quand il sort du bas vers là-bas, alors il marque le beau temps; par contre quand il fait pli pli pli vers le bas, quand il descend vers les vallées, alors c'est le contraire, le pivert vers le bas pli pli pli, la pluie est certaine.

Je n'examine pas dans le détail les onomatopées des bruits prêtés au divers animaux mais, je fais remarquer qu'à Arbouet-Sussaute, le témoin, parfait bascophone et gascophone, signale un autre oiseau dont le mouvement est représenté par "plau plau plau plau!":

Baduzü mota bat beno geiho eh! Baduzü xuria e, hua khasü, denborak khanbiatü nahi dizi hua igaiten delaik aphal aphala lürrain arrasetik, plau! plau! plau! plau!... "ilháintxuri", le milan blanc...

Vous avez plus d'une sorte (de rapaces) hein! Vous avez le blanc, alors lui attention, le temps veut changer quand il passe au ras du sol, plau plau plau plau, le milan blanc...

Or ce plau évoque en gascon l'eau et la boue. Palay dans son dictionnaire indique : Onomatopée d'un coup sur l'eau, la boue, une chose molle, plaf".

D'autre part, et toujours au chapitre des relations avec le gascon, Henriette Etcheverry Mendilahatxou, notre témoin de Labastide-Clairence, parfaite gascophone, cite le gascon escoute-plouye, non pas comme le nom du pivert, mais en emploi métaphorique, pour désigner quelqu'un qui ne tient pas sa langue et qui est source de disputes en rapportant ailleurs ce qu'il a entendu ici. Elle est consciente cependant que l'expression ne peut être traduite en basque. Selon les témoins basques gascophones du pays de Mixe, le gascon utiliserait gay, le 'geai', et non pas la désignation du pivert, pour stigmatiser un individu, emploi métaphorique que Palay ne donne pas. Par contre Palay atteste escoute-plouye dans un sens proche de celui de Labastide: "Ecoute-pluie, qui est dans l'attente ; un distrait, quelqu'un qui semble toujours écouter s'il n'arrive pas quelque chose, ou quelqu'un ; un espion ; un curieux indiscret et qui tâche de n'en avoir pas l'air".

Après cette digression provoquée par ces rapprochements possibles avec le gascon voisin, j'en reviens aux proverbes qui ont été recueillis sur le pivert annonciateur de pluie.

Busturia : Okille egarri de ta eurixe in biedau.

Le pivert a soif et il doit pleuvoir.

Berriz : Leenago sárrak esate ebién "irú egúnbarrú eurie... okille kantári daonien irú egún barrí eurie"...

Autrefois les vieux disaient, dans un délai de troi:: jours la pluie, quand le pivert est en train de chanter, dans un délai de trois jours la pluie.

Beasain : Ebi eske eote'men dala ... earrik eo, a a! okille ko-ko-ko-ko! inez ta "okille ebi eske zeok, egarrittu izango ek!" esan oi da...

Le pivert est dit-on en train de réclamer, il aura soif ou bien... en faisant ko ko ko ko et : le pivert est en train de réclamer, il doit avoir soif!

Arrast-Larrebieu : Úkhüla errekan, harrezak kapixüna kaallan, ükhüla errekan, markha ebia horra dela. 
Si le pivert va vers la vallée, détache ton manteau de la cheville (à laquelle il est suspendu au mur); si le pivert va vers la vallée, c'est la marque que la pluie arrive.

J'ai dit que l'oiseau était généralement annonciateur de pluie. Outre celles rapportées plus haut provenant isolément de Domezain ou Baïgorry, les informations recueillies semblent dessiner en Labourd côtier une aire dans le bassin de la Nivelle où les témoins laissent entendre que l'oiseau marque la sécheresse. Mais on ne sera pas surpris de cette inversion entre pluie et sécheresse si on veut bien admettre que l'opposition est aussi une forme de contiguïté. À Hendaye est manifeste un désaccord entre générations sur l'interprétation à donner à la présence de l'oiseau.

Hendaye : Gue aita zenak esaten zuen, ori kantai, ebiya galdez aitzen zela... to ! ura galdezka ai da ! eta nik jenealin... idortia sumatu izatu ut...

Notre défunt père disait, le pivert est en train de chanter, tiens il est en train de réclamer de l'eau, et moi en général j'ai plutôt remarqué de la sécheresse...

Saint-Jean-de-Luz : Erraiten dute kantari delaik idortea. Arraro da orai.

On dit que quand il chante c'est la sécheresse.

Les témoins de Sare et Saint-Pée-sur-Nivelle confirment la même information.

Arcangues : Errate'úte idor, dénbora edérra delaik aitzen dela kantuz...

On dit, que c'est le temps sec, le beau temps quand on l'entend chanter.

Selon les témoignages suivants, l'oiseau annonciateur n'est pas le pivert. $C_{\epsilon}$ peut être la pie : la hauteur à laquelle elle dispose son nid dans les arbres est censé indiquer si la saison est pluvieuse ou non, et ce selon plusieurs informateurs. Nous ne donnons qu'un exemple, celui d'Arhansus, parmi bien d'autres.

Arhansus : Okhiloa eztiakizut bena xoriek, phikek ba, kafiak, kafia, phika, ohantzia nola erten duzie ziek? Hek gora iten duztelaik arboletan ordian bustite anunzatzen eta hek behera in duztelaik idorte, phikak, ithaxuren gatik behera in eta, gañeko ithaxura h:ldu bitira hori señalia, ba, hanitzek erten zi ien, bo berrikitan e, xaharrek eta: "To! aurthen idorte izain duk eta phikak aphal iten tik kafiak".

Le pivert je ne sais pas mais les oiseaux, les pies oui, les nids, "kafia" le nid, "ohantzia" comment dites-vous vous autres (gens de la côte)? Quand les pies font leur nid très haut dans les arbres alors elles annoncent une saison très pluvieuse, et quand elles les font très bas, c'est la sécheresse, les pies, à cause des gouttières, elles le font plus bas, parce que les gouttières se produisent, çela est un signal, oui, beaucoup le disent, récemment encore, les vieux et: "Tiens! Cette année on aura de la sécheresse, la pie fait les nids très bas".

Ailleurs ce peut être la buse (avec un emploi métaphorique de l'expression haize phikatzen 'faucher le vent').

Bunus : Buzoka, ba, ba mendi gañ hoitan egoiten tzu geldi-geldia, buzokak, buzoka aire phikatzen ari uk hantxe, bi egunen buruko euria, ba.

I: Haize pikatzen ez da auher izaitea? 
$L$ : Ah ba, erten duzu ba hori, gizon bat lanian, laneat joan eta beha egoiten balinbada, ze ari da? E haize phikatzen ba ba, edo hola, auher, erten hoi e, ta buzoka hua re haize phikatzen ari, airian.

La buse, oui, oui, elle reste sur ces hauts de montagnes sans bouger, la buse, la buse est en train de faucher l'air, au bout de deux jours c'est la pluie, certes.

E : Faucher l'air, cela ne veut pas dire rester à ne rien faire?

$T$ : Ah oui, cela aussi se dit, un homme au travail, il va travailler et il reste là à regarder, qu'est ce qu'il fait? Ah il fauche le vent, oui oui ou bien ainsi, paresseux, cela se dit aussi, et la buse aussi est en train de faucher le vent, dans l'air.

\section{Le milan et d'autres rapaces reviennent souvent dans les témoignages.}

Bardos : Mülüxuriak, hak badu hegaldatzeko manera itxasoko alderdeat iten bazen, ordian uria zen, bai fite, eta hala hala bestalde iten bazen bena etzen bestalde usu ikhusiko, denik e euria zelaik bethi haat itxasoari buruz iten zen hegaldatzen, lekhian gainian.

Le milan blanc, il a une manière particulière de voler, s'il le faisait du côté de l'océan alors c'était la pluie, et de la même manière s'il le faisait dans l'autre sens, mais dans l'autre sens on ne le voyait pas souvent, à tout prendre, la pluie était là quand il volait tourné vers l'océan, sur place.

Domezain : Agorrilan hüxtüka ari deluik, ebia galdeiten diela erten dizie, miiii miii ari bita, entzuten eta hura galditen diela erten dizie. - Amak : Ba, etzi edaten ahal euria iten delcik bezik ordian. - I : Jinkoak puniturik? - C L : [irriz] Ah berdin! etzit entzün, iten dü hüxtü bat, hiii...

En août quand il est en train de crier, on dit qu'il réclame la pluie, il fait mii miiii, on l'entend et alors on dit qu'il réclame de l'eau... Oui il ne peut pas boire d'autre eau que l'eau de pluie alors dit-on. E : Puni par Dieu? T: Ah tout aussi bien (riant), je ne l'ai pas entendu, mais il fait un cri, hiiii...

Larrau : Ülhaintxak e badizü büiltaka aitzen tützü lekhün bein aide hartzen, ordin haik e denboa khanbio, frantsesez erraiten beita sur place egiten hola, haik e erraiten zizien baziela, ; gio erran den, gue herri aizo hortan (Senta Garazi) deitzen dizie behibidetako andexuia, gük ez, saixuia edo gük bestela hegaxti tous les oiseaux, lümakuk, txipieneik handienila, ordin hegaxti hoik oo saiak, ordin saixui, saibeltza eta saiak, been...

Le milan aussi, quand vous les voyez parfois faire du surplace comme prenant le vent, alors eux aussi annoncent changement de temps. Ensuite, le dénommé, dans le village voisin (Sainte-Engrâce), ils appellent un oiseau du nom de la dame blanche des chemins de vaches, nous non, nous l'appelons vautour blanc, et sinon nous appelons hegaxti tous les oiseaux, tous les animaux à plume du plus petit au plus grand, alors sont hegaxti tous ces animaux, le vautour blanc, le vautour noir, leur...

Ordiarp : Gio bada hau ere, ("Busard Saint-Martin", hoik eta biak süstut, ezta negian egoiten eta ebia espeantxa dielaik aphal aphala ebilten dia eta gio ehaisten zerbait badelaik atzemaiteko, kholpia segür die horiek. 
Ensuite il y a aussi celui-ci (le busard Saint-Martin, selon Peterson), ceux-ci aussi et les deux surtout, il ne reste pas ici en hiver et quand il attend la pluie ils volent très très bas et ils descendent aussi pour attraper quelque chose, leur coup est très sûr.

Ordiarp signale aussi l'épervier.

Ordiarp : I : Zoin da denbora kanbio markatzen duena? L : Esparbea, lekhian gainian hola ai beita. Esparbea.

E: Quel est celui qui annonce le changement de temps? T : L'épervier, et qui reste ainsi sur place. L'épervier.

Ordiarp : Airian mugitü gabe, esparbeak egiten dik ene uste, aidik phezatzen dik, ze denboa den.

Celui qui fait du surplace, c'est l'épervier qui fait cela je crois, il pèse le temps et quel temps il fait.

Ordiarp : Hunek e pasajia egiten dü, sasu huntan ikhusten da eta ürzuak lotsa dira izigarri horrentako. Erraiten die augaitza expaantxa diela, nik eztakit zer den, haiziai bürüz, haizik nuntik buhatzen beitü, eta hai bürüz egoiten, aitzen da lekhün gainian; atx! horrek augaitza dik, aizephikatzen hegaltatzen ibili gabe, aizepikatzia erran nahi da, gizun batek denboa igaiten badü deusik lanik agertï gabe, horrek aize pikatzen eantsi dik egün, ezpadü lanik agertü.

Celui-ci il a son passage, en cette saison on le voit et les palombes ont terriblement peur de lui. On dit qu'il annonce la tempête, je ne sais pas ce que c'est, il fait front au vent, il fait face à la direction d'où souffle le vent, il fauche le vent, sans donner un coup d'aile, faucher le vent, c'est-à-dire, si un homme passe le temps sans qu'on voit de travail qui soit fait, oh il a passé la journée à faucher le vent, s'il n'a pas montré de travail fait.

A Larrau et Sainte-Engrâce on cite le pernocptère.

Larrau : Eta behien bideko emazte xuia, hoi Senta Gaazin, gük eztü erraiten, martxuan agertzen balinbada, arraützültzen bada negü den lekhütik markha hun da, bedatsia zabaltzen dela, koblaka dira ordenaioski.

Et la dame blanche du chemin des vaches ('le pernocptère'), c'est l'appellation à Sainte-Engrâce, nous ne le disons pas; mais quand il apparaît en mars, s'il revient ici, à partir du moment où il cist là, c'est bon signe, et que le printemps va s'ouvrir, ils sont par couples en général.

Sainte-Engrâce : Andexuia, jiten dük denboaen huntziai, bat jiten dük lehenik, etauano bia jiten denian, denboa huna markhatzen die... Eztüzü gaxtua hua, eztizü olloik jaten ez txitxaik.

La dame blanche ('pernocpière'), elle vient quand le temps se met au beau, l'un vient tout d'abord, et quand l'autre vient aussi, ils annoncent le beau temps... Elle n'est pas méchante elle, elle ne mange ni poule ni poussin.

Montory : Aphobelatza, aphobelatzak egiten dik hüxtü bat, la buse erraiten dizie agorrilan egarri dela eta ebia nahi diela ekharraazi. 
La buse, la buse produit un sifflement, on dit que la buse en août a soif et qu'elle veut faire venir la pluie.

À Behorléguy l'oiseau n'a pas été identifié.

Béhorléguy : Eurieskalia, eztakit zoin den... hegi batian egoin tzu higitu gabe, holaxet hegala fla-fla-fla... ta geo sartuko da behiti ta berriz e inen du inguru bat, kusten tzu ta "ya! Euria eztuk urrun!", euri-eskalia... xuxen zoin den, ezta buzoka, hoietaa tiatzen dien zeait duzu... ttipixioño...

Le quêteur de pluie, je ne sais pas lequel est-ce? Ils restent sur une crête sans bouger, comme cela avec l'aile qui fait fla fla fla, et ensuite il descendra et fera de nouveau un autre tour, vous les voyez et : Aie! Tu n'as pas la pluie bien loin! le quêteur de pluie, exactement lequel ce peut être? Pas la buse, mais un oiseau qui tire sur les buses (leur ressemble), mais plus petit.

La corneille est aussi citée.

Larveveau : Belatxa, eztiakizut, hola geldi geldia lekhian ari den bauzu zonbaitaldi, bena hua denbora txaretan, hua geldi geldia ari dena.

I : Zer erran nahi du? euria nahi duela edo.

L : Bo! eztiakizut nik, to denbora khanbiatuko! Airephikatzailia ari duk, aire phikatzen, airia, holaxe lekhian ari dena, nik etzit behin jeus e intzun.

La corneille, je ne sais pas, comme cela vous la voyez parfois qui reste sur place parfois, mais celle-là quand il va faire mauvais temps, elle reste à voler sur place. E : Qu'est-ce que cela veut dire? Qu'elle veut la pluie ou bien? T : Oh je ne sais pas moi, tiens le temps va changer! Le faucheur d'air est en train, il est en train de faucher l'air, l'air, comme cela sur place, oui je n'ai jamais rien entendu.

Domezain : B lekukoak: Miriak edo ilhaintxak iten dik haizia pikhatzen. - C lekukoak: Ah! hori belatxa, hola lekhiain gainian, eta hola geldi geldi, üria markhatzen dü horrek, hola ari delaik, bi egün gabe eüria eh! eta hola ta blup behero ta berdin orenak

Témoin B : Le milan il fauche le vent. Témoin B: Ah mais il s'agit alors de la corneille, en train de faire du surplace, et comme cela sans bouger, elle marque la pluie, quand elle est en train de faire cela, au bout de deux jours la pluie! Et ainsi et puis blup! Elle va plus bas et continue ainsi des heures durant.

Dans une dernière partie je donne le texte des narrations recueillies ou les passages qui laissent deviner l'existence d'un récit plus complet que le locuteur ne donne pas entièrement. Comme ci-dessus, dans certaines narrations, le protagoniste n'est pas le pivert mais un rapace ou un corvidé.

Les narrations souletines ont souvent pour protagoniste le milan ou un autre rapace.

Larrau : Gio ilhaintxake, egarri, zerbait, Jinko huna etziela obeditü, eta gio eman ziela üztailagorrilak hurik gabe egoiteko, hua gaztigü bezala, bestelan ükhüla ai balinbada nabetan pil pilpilka ordin denboa xüxen txarrilat...

Puis le milan, il a soif, quelque chose, il n'aurait pas obéi au Bon Dieu, et ensuite qu'il lui avait ordonné de passer les mois de juillet et août sans eau, et 
cela comme châtiment, sinon quand on voit le pivert descendre vers la vallée en faisant pil pil pil, alors le temps va tout droit à la tempête.

Larrau : Üztail-agorriletan etzitia beste lagünak lagüntü, pünitïik izan zela, üztail-agorriletan hurik etziela edaten ahal, hurik ezin edanez egiten ziela hüxtü hoi, ilhantxak, arraprua bazen iilhaintxak bi ilabethez Jinkuak pünitü ziela etzilakotz behar bezala zerbeit egin.

En juillet et août, il n'avait pas voulu aider ses compagnons, et qu'il avait été puni, qu'il ne pouvait pas boire une goutte d'eau en juillet et août, et que ne pouvant pas boire d'eau il produisait ce sifflement, le milan, le dicton existait selon lequel pendant deux mois Dieu l'avait puni parce qu'il n'avait pas fait quelque chose comme il le fallait.

Esquiule-Féas : Ükhüla nabetaik jin nahi delaik, eztakit zuñ erran nahi diin, ebia nahi dielaik zeen gañen nun e beita idorregi dela erran nahi lüken eztakit. entzün dit ülhaintxaz ba, jüsto jüsto ez, Jinkuk galthatü ziela zerbaiten egitia eta errefusatü ziela eta ordin hartakotz urtheko bi ilabethe beoenetan erran nahi beitü üztaila eta agorrilan, amañi zenak erraiten zien, bethi ülhaintxak ai ziela bethi bata bestiai oihüz, eta geo ta goago juiten ziela egarriz, hala erraiten zikien ; geo...

Quand le pivert veut venir de la plaine, je ne sais pas ce que cela veut dire, qu'il veut la pluie parce que certainement sur les hauteurs il fait trop sec, est-ce cela qu'il voudrait dire? Je ne sais, je l'ai entendu au sujet du milan, pas très exactement c'rtes, que Dieu lui avait demandé qu'il fit quelque chose et qu'il avait refusé et qu'alors c'est pour cela que durant les deux mois les plus chauds de l'année, c'est-à-dire en juillet et août, ma défunte grand-mère disait, qu' les milans criaient de l'un à l'autre et qu'ils allaient de plus en plus haut sous l'empirc de la soif, on nous le racontait ainsi, après...

Je dois au témoin de Alçay la narration souletine la plus formelle.

Alçay : Ez, aphobelatzak bai; hua entzün düzia zentako kurritzen aitzen den... Gisala manhatü zizün behin aska bat beitzen zihkin, huraintako xahatzea, hoi leheneko erran xaharra entzün dit. Aphobelatzak xaha lezan hua Jinko Hunak manhatï zizün eta izigarriko azpi ederrak titzü hak, holli holli holliak. Lotsa züzün zikhint. Etzizün e hunki nahi izan. Loliak egin zizün hak ezpeitzian zikhintzia arriskatzen; nik e entzütia dit hola eta Jinko Hunak erran ziozün: "hik üztailagorriletan bethi kwik kwik" errekondo hoien gainti. Lehenoko xaharrer dit. entzün artzañ xahar eli bati. Hek hala khuntatzen ziküzien gü haur ginelaik borthian, erran hoi

Non, la buse oui, celle-là avez-vous entendu pourquoi elle est toujours en train de circuler? Paraît-il on hui avait ordonné, étant donné qu'une auge était très sale, de la nettoyer. Ceci est un vieux dit quej'ai entendu. Le Bon Dieu avait commandé à la buse qu'elle nettoyât cette auge, mais la buse a des cuisses très belles, très très très jaunes. Elle avait peur qu'elle ne se salît. Elle n'avait pas même voulu toucher à l'auge. Le corbeau quant à lui il l'avait fait parce qu'il ne risquait pas de se salir; moi aussi je l'ai entendu comme cela et le Bon Dieu lui avait dit: "Toi en juillet et août tu seras toujours à faire kwik kwik", tout au long 
de ces bas de vallées. Je l'ai entendu dire aux anciens, à beaucoup de vieux bergers. Eux nous le contaient ainsi quand nous étions enfants, en estive, ce dit...

En Navarre Koro Segurola et Izaskun Etxebeste ont recueilli des versions dont le corbeau est protagoniste malheureux alors que dans la version de Alçay c'est lui qui ne craint pas de se salir en nettoyant la fontaine.

Beruete (Navarre) ; Ok esaten duté, bi illebetéz, úrik eztutéla eán bear ; emén dute kastigu pátu bele ré, bi illebetéz : júnio eta abriztué, júlio ta abúztué, úrik éztute (e)án bear..., ezín dutelakó ; eátekótan eán beuté (behar dute) árbolan éittem báita intzé, intzué txupátu... Démbo atén, pátu zitxióten kástigu izáan da; démbo atien esaten zén, kástiguk aziéndái ta emáten zizkiótela ; olákon bat izáan da, go atték, béte (beti) ói esáten zun... Arek esáten zun : "belék zalápartaka dáaltzé, ta zér dú ? ta, úrik ezpaitézake...

On dit que pendant deux mois, ils ne doivent pas boire d'eau; les corbeaux devraient supporter un châtiment, pendant deux mois, juin et août, juillet et août, ils ne doivent pas boire d'eau, ils ne peuvent le faire. Tant qu'à boire, ils ne peuvent boire que la rosée qui se dépose sur les arbres, ils sucent la rosée. A une époque, on leur a donné un châtiment, à une époque, on disait que des châtiments étaient donnés aux bêtes. On dit : les corbeaux se déplacent à grand bruit, qu'ont-ils donc? C'est qu'ils ne peuvent avoir d'eau.

À Sumbilla (Navarre) un récit qui a aussi pour protagoniste le corbeau retrace les mêmes faits : le corbeau n'a pas l'autorisation de boire durant deux mois ; le corbeau est puni par Dieu pour n'avoir pas aidé au travail ses compagnons.

À Echalar enfin, c'est le faucon qui est le protagoniste.

Echalar (Navarre) - A lek : Ixtori ori zen, \%San Pedro ta \%Jaungoikua, biyek bazabiltzan mundu mundu ta egarritu eta asi zien erreka batin ura edaten ta sapeltsa batek ura in zion zikindu ta orrengatik sapeltsa egoiten da bi illautez urik edan gabe. - B lek : Eta errekan ementxe ibiltzen die bueltan beti. - A lek : Beti kuiii! kuiii! ta errekan ibiltzen die... Bakarrik libre te abre ferratu bat, zaldi furratu bat o mando ferratu bat, aren ferran, ura balin bada ferra artan, artan bakarri(k) te libre... zulu artan balin bada ura artan bakarrik du libre [irriz]...

L'histoire était ainsi, Saint Pierre et Dieu, les deux allaient de par le monde et ils étaient très assoiffés et ils avaient commencé à boire l'eau d'un ruisseau et le faucon leur avait sali l'eau et alors il doit rester durant deux mois sans boire. - Témoin B: Et on le voit qui ne cesse de tourner au-dessus du ruisseau. - Témoin $\mathrm{A}$ : Et kwui kwui ils vont et viennent près de la rivière. Ils ont seulement l'autorisation, vous savez une bête ferrée, un cheval ferré, ou un mulet ferré, dans son fer, s'il y a de l'eau dans son fer, il peut boire cette eau, s'il y a de l'eau dans ce fer ils peuvent en boire (on sait que le fer à cheval, généralement percé, n'est pas un récipient idéal pour y boire quoi que ce soit).

Azkue, dans son riche recueil de 1935, rapporte aussi des informations qui établissent un lien entre l'eau et les oiseaux bele, le 'corbeau' et erroi (le 'grand corbeau' ou le 'freux'?). Ces textes n'étant pas connus en français j'en donne aussi la traduction après le texte basque recueilli par Azkue. 
Barcus : Behin xori-kunte guziak üthürri baten xahatzen ari ziren. Ama birjinaren üthürria zen. Ordüan erroia horra da. Besteak (sic) erraiten dakote : guk bezala hik ere xahezak. Erroiak erran zien : xankoak ederregi ditiat hor erabiltzeko. Ordüan Yinko Hunak erran zakon : bi ilabetez, uztailan ta agorrilan, eztuk hruik edan ahal izanen; eta egarriz marrakaz ariko hiz (S-bark)

Une fois la gent des oiseaux était en train de nettoyer une fontaine. C'était la Fontaine de la Sainte Vierge. Arrive alors le freux. Les autres lui disent : toi aussi fais comme nous et nettoir-la! Mais le freux leur dit: j'ai les pattes trop belles pour les salir à tâche pareille. Alors le Bon Dieu lui dit : pendant deux mois, en juillet et août tu ne pourras pas boire d'eau, et tu seras en train de hurler à cause de la soif (Azkue, 1935, I, 108).

Dans le récit suivant collecté aussi par Azkue, le récit explique pourquoi le freux est noir.

Vidangoz : Noeren kutxako erroia zuria emon zen. Yaungoikoak bidali ta utzuli etzenean madarikatu egin zion, kaur erranez : zuri izan yaz, beltz ut uliren yaz, garagarzaroan ta gari-ielan urik eztuk edateen. Ilebete korietan ura edan balezate, garagarra ta garia elokekete ezaguturen.

On dit que le freux, dans l'arche de Noé, était blanc. Dieu l'avait envové aux nouvelles et comme il ne revint pas, Dieu le maudit en disant ceci : Tu as été blanc, et tu deviendras noir et en juin (litt 'mois de l'orge') et en juillet (litt. 'mois du blé') tu ne boiras pas d'eau. Et si jamais les freux buvaient de l'eau durant ces deux mois-là, les hommes ne connaîtraient ni orge ni blé (Azkue, 1935, 107).

On ne sera pas surpris que le récit dans lequel le pivert soit cité apparaisse aussi dans Azkue qui en donne une seule version recueillie en Guipuscoa.

Jaungoikoak txori-mota guztietatik bilduarazi ta esan zien; "Guztion artean iturri bat egin ezazute". Okilak etzuela berak iturri-bearrik esan zuen, goitik zetorren ura edango zuela-ta. Arrezkero, legortea danean euri-eske lur-kur-kur ari izaten da okila.

Dieu dit un jour aux oiseaux dont il avait réuni toutes les espèces: "Entre vous tous, faites une fontaine". Le pivert dit qu'il n'avait pas quant à lui besoin de fontaine, puisqu'il boirait de l'eau venue d'en haut. Depuis lors, quand il fait de la sécheresse, le pivert est en train de réclamer de l'eau en faisant kur kur kur (Azkue, $1935 ; 112$ ).

On sait que Rolland 1887 cite aussi le corbeau :

Dans la Carinthie, ce sont les corbeaux qui, par punition sans doute de quelque méfait, n'ont droit pour se désaltérer qu'à l'eau de pluie.

Detienne et Vernant 1974, 204 citent pour la Grèce l'oiseau appelé aithuia qu'ils traduisent par 'corneille de mer', mais qui pourrait être le puffin, le grèbe... animal censé conjoindre ciel et eau et relier eau et terre.

Après cet emprunt aux données de R. M. Azkue et du domaine européen, je reviens aux narrations que $\mathrm{j}$ 'ai recueillies et dont le pivert est le protagoniste.

Je n'ai pas d'explication au changement de protagoniste entre rapace et pivert. J'ajoute cependant au dossier une remarque curieuse de J. de Pesquidoux, excellent connaisseur de la ruralité gasconne du début du siècle, qui dans un chapitre 
de sa série Chez nous (la référence exacte m'échappe) raconte comment l'épervier semble avoir pour victime de prédilection le... pivert sur lequel il s'acharne en lui fracassant le crâne à coup de bec !

Je redonne ici la narration de Juxue avec le pivert pour héros et que le lecteur a lue en exergue. Je remercie ici le témoin, M. Pierre Pétotéguy, qui m’a mis sur la piste de ces récits.

Juxue : Erten da, "to okhiloa ai duk arbolari kokha, okhilia egarri, euri inen dik!". Okhiloak etziela nahi putziain xahatzen ari eta gio okhiloa egarri zela eta edaten zien gisala ostoetarik erori ura, euria ari zelaik bakharrik eta koskaka ari delaik arbolari ; "to egarri duk! Euri nahi fite!". Okhiloak zangoak biziki garbi ditu : etzielakotz nahi putziain xahatzen ari. Eta gio ahatik egarri ederrik pasatzen. Erten, erten. "Eztuk nahi zikhindu, egarriaekilan egoin hiz!".

On le dit: "Tiens le pivert est en train de cogner dans l'arbre, le pivert a soif, il va pleuvoir". Que le pivert n'avait pas voulu nettoyer la mare et puis que le pivert était assoiffé et qu'il buvait seulement, parait-il, l'eau tombée des feuilles, et quand il cogne l'arbre: "Tiens il a soif! Il veut pleuvoir!". Le pivert a les pattes très propres. C'est qu'il ne voulait pas nettoyer la mare. Et ensuite cependant il passe par de belles soifs! On le dit, on le dit. "Tu "i'as pas voulu te salir, tu resteras assoiffe".

Hosta : Ah nik intzun dudan historioa okhiloaina uzu. Okhiloak eztautzu ithurri batian edaten ahal gisala, oai okhiloak, errekan eta ithurri batian eztautzu edaten ahal, nik entzun dudana, gin eztiakizut zeen hori heen, egia ala gezurra den, baizik eta galdein zutela ithurriain iteat ihize(n), leheno, erran bat duzu, eta ez omen zuzun joan, eta gioztik gisala euria iten dielaik, ihitzian edaten omen dizi, gio?

\section{I: Entzun duzu?}

$L$ : Intzun dizit ba, intzun... Ez ?, Ba ba eta okhiloa marrakaz ari delaik, marrakaz ari bitu, egarria dela erten dizie, euria galdeiten diela... Nik intzun dit hori erten lehengo xaharrer, iturri iteat galdein zutela eta etzela, etziela joan nahi ukhan, gio ? Egia ala gezurra da? Eta ordian etziela errekan ez ithurrian edaten ahal, behar diela ihitza edateko.

Ah l'histoire que j'ai entendue, celle du pivert, c'est celle-ci. Le pivert ne peut pas boire, dit-on, dans une fontaine, maintenant, le pivert ne peut pas boire dans le ruisseau ou dans la fontaine, c'est ce que j'ai entendu, après je ne sais pas parce que..., est-ce vrai ou faux? Comme quoi on leur avait demandé de nettoyer la fontaine des animaux, c'est un dit d'autrefois, et dit-on, il n'y était pas allé, et depuis lors, dit-on, quand il pleut, il ne peut boire que de la rosée, après?

E: Vous l'avez entendu?

$T$ : Je l'ai entendu, entendu... Non? Oui oui et quand le pivert est en train de crier, parce qu'il crie, on dit qu'il a soif, qu'il réclame la pluie... Moi je l'ai entendu dire aux anciens autrefois, qu'on avait demandé d'aller nettoyer la fontaine et qu'il n'avait pas voulu y aller, après? Est-ce vrai ou faux? Et alors qu'il ne peut pas boire dans le ruisseau ni dans la fontaine, qu'il lui faut de la rosée pour qu'il boive. 


\section{Note sur les oiseaux auxiliaires de la justice}

Bien qu'il soit distinct des récits sur l'oiseau et la pluie, j'ajoute ici un récit recueilli à Aussurucq et que je veux offrir à Jean Haritschelhar puisque M. et Mme Jaragoyhen me l'ont fourni alors que je pensais enquêter sur la réception du poète souletin Etxahun, sujet de la thèse de Jean Haritschelhar. Le texte peut paraitre confus et exige quelques explications sur ses conditions d'apparition.

Les témoins d'Aussurucq, au riche savoir empirique et bons connaisseurs de la tradition orale, venaient de m'expliquer que les thèmes de certaines chansons de Pierre Topet-Etxahun n'avaient pas perdu toute actualité : à preuve, un incident qui se produisit lors de noces en Soule au début du siècle. Comme c'était l'habitude, chaque invité aux noces devait "donner" une chanson devant toute l'assistance. Mais lorsqu' un convive commença à entonner une chanson d'Etchahun intitulée Amodiogati, toute une famille attablée à ses côtés se leva d'un bloc et quitta les lieux. Il s'agissait de gens qui s'étaient sentis offensés par le fait que cette chanson soit reprise, alors qu'elle fait allusion à un assassinat survenu en 1854 dont un membre de leur famille passait pour le présumé coupable. J. Haritschelhar donne le détail de la chanson et du fait divers qui lui a servi de point de départ (Haritschelhar 1970, 638-649). Ceci explique les noms de famille cités dans le texte ci-dessous au début et qui font allusion aux personnages cités dans la chanson.

Mais après avoir cité cet événement, les témoins d'Aussurucq passent sans transition marquée à un autre récit que j'ai pris d'abord pour un autre fait divers survenu en Soule à une date indéterminée certes mais pas forcément très éloignée. C'était de ma part négliger la capacité que le mythe a de s'enraciner dans un espace et un temps qui est celui de l'énonciateur. En effet, quelle ne fut pas ma surprise de constater que ce récit dans lequel les grues se font auxiliaires de la justice n'est pas inconnu de la littérature populaire ni même de la littérature savante, puisque l'écrivain latin Ausone rapporte le fait comme tiré d'une légende latine liée au poète Ibycus.

Mais voici d'abord la transcription du texte d'Aussurucq (on pourra en entendre la version orale sur CD audio dans l'anthologie de documents tirés de EHHA publiés par Euskaltzaindia, à paraître, puisque j'ai choisi cet extrait, difficile d'accès certes, comme représentatif du basque d'Aussurucq, outre son contenu qui nous intéresse ici : le linguiste "pur" y relèvera par exemple dans zi(r)ateke le futur synthétique endémique à la Soule).

LI. : Badakizie nunti atzaman zien hura? hil...

L2. : Ez hoi, ez hoi hoi hoi jin ziozün geo, beste bat..

LI. : Ah! Gaindañeko istoia eta...

L2. : Ez, ez, ez, Etxandin eta Iratxabalen geo, hack atzeman zizien..

L1. : Ba bena beste batek atzaman zizün..

L2. : Ah bai...

LI. : Gaindañen züzün hua eta zea...

L2. : Zük erran nahi, zuñ erran nahi düzü?

L1. : Hil abantzü zen gizona eta khürloak igaiten ziren gañen eta erran zon: - Zik ene jakile zirateke! eta hantik urthiaen bürian boogatzen tü ehuak igaiten eta :- Bazuzte zeaen, haik ehuan, haen jakilik bazuzte oai, erran zin eta hartaik atzaman zien 
X. : Nola nola nola? eztut...

L2. : Soizü : eho zizin batek bestia...

X. : Garindañen...

L2. : Gaindañene eta hil beh...

L1. : Hiltzen ari zenak...

L2. : Hiltzen ai zenak erran zizün khürluk igaiten beitziren : - Zik ziateke ene jakile ! eta geo hantik eta urthiaen bürian arra entzün zitzün eta geo : Khürluak entzüten tützie?

Eta hiltzale haek : - Bai to badutzak zeaen jakilik!

L1. : Bai...

X. : Bere buria saldu?

L2. : Bee büia..

L1. : Bee büia salatü zin hartaik.

T1. : Savez-vous de quelle manière on le trouva celui-là ? L'assassin?

T2. : Non, mais ceci, ceci, ceci survint ensuite, un autre...

T1. : Ah l'histoire de Garindein...

T2. : Non, non non, pas Etchandi et Iratchabal, celui-là on l'avait attrapé.

T1. : Oui mais un autre l'avait attrapé...

T2. : Ah oui...

TI. : Il était à Garindein quand a luí.

T2. : Vous, vous voulez, vous voulez parler de...?

T1. : L'homme était à demi-mort, et les grues étaient en train de passer au même moment, et il leur avait dit : Vous serez mes témoins! Et puis au bout d'un an, cet assassin-là entend passer les grues et il leur dit : - Vous partez, les grues - à leur bruit -, et vous, les témoins d'un tel l'homme assassiné), vous partez maintenant, dit-il, et par cela il fut attrapé

$E$ : Comment, comment? comment? Je n'ai pas compris...

T2: Faites attention : un homme en avait tué un autre...

E: A Garindein...

TI : À Garindein celui qui devait mourir..

T1: Celui qui était en train de mourir...

T2: Celui qui était en train de mourir avait dit, étant donné que les grues passaient : Vous serez mes témoins! Et puis après cet événement et au bout d'un an en les entendant à nouveau l'assassin avait dit : Entendezvous les grues? Tiens ce sont les témoins d'un tel qui s'en vont!

TI: Oui...

E : Il s'était trahi...

T2: Lui-même.

TI: Il s'était trahi par cette phrase.

Ce texte dont le récit lui-même n'occupe que quelques lignes, récit que j'ai fait répéter faute de l'avoir immédiatement compris, est connu par exemple dans la tradition catalane. Il a été recueilli à Barcelone en 1914 par Joan Amades 1950, J'en donne le texte d'après la traduction en français de Marlène Albert-Llorca apud Amades 1988, 114. L'économie en est un peu différente du texte d'Aussurucq puisque l'assassin ne se trahit pas lui-même par forfanterie. 


\section{Ce que disent les grues}

Voilà qu'une fois, un homme appelé Martin fut tué dans un endroit désert. Personne ne vit le crime, sinon les grues qui étaient en route, et la justice chercha en vain le coupable? Celui-ci se disait.

Ils peuvent toujours chercher! Personne n'a rien vu, à part les grues; mais elles étaient en route, et qui sait où elles sont maintenant!

L'année suivante, cependant, les grues revinrent et les oiseaux leur racontèrent ce qui s'était passé. Alors les grues se jurèrent de chercher le criminel et de le dénoncer à la justice, puisqu'il osait se moquer de leur silence. Elles le cherchèrent partout et trouvèrent bientôt dans un village où l'on dansait pour la fête. Elles se mirent aussitôt à voler sans arrêt au-déssus de lui en criant :
- Es aquí, és aquí
Il est ici, il est ici,
el que va matar celui qui a tué
En Marti
Assassi, assasí. le Martin Assassin, assassin.

Et aujourd'hui encore elles crient la même chose en manière d'avertissement : que les gens ne croient pas surtout pas qu'ils peuvent compter sur le silence des oiseaux et, si les oiseaux n'ont encore rien dit, qu'ils ne s'avisent pas d'en dire du mal (Joan Parcerisses, Barcelona, 1914)! Je ne peux que citer la note de Marlène Albert-Llorca qui suit ce récit :

"Le motif de ce récit est repris d'une légende latine liée au poète Ibycus : celui-ci périt assassiné, et le crime aurait été dénoncé par les grues (cf. Ausone, Technopoegnie, X, 77, Euvres en vers et en prose, T. I, Librairie Garnier, Paris, 1934)".

Dans cette contribution à un hommage en l'honneur de Jean Haritschelhar dont la thèse a été essentielle pour à greffer à nouveau la tradition orale et les œuvres d'Etchahun dans la communauté souletine, je livre à la sagacité du lecteur cette narration "populaire" d'Aussurucg comme résurgence ou écho lointain (à quinze siècles de distance) d'un récit déjà signalé par cet écrivain aquitain du $\mathrm{IV}^{\mathrm{e}}$ siècle que fut Ausone.

Mes remerciements vont à tous les témoins et locuteurs dont le savoir et la patience ont permis ce travail.

X. VIDEGAIN

UMR 5478, UPPA

1. EFHA (Euskal Herriko Atlas Linguistikoa) est un projet de longue haleine mené par Euskaltzaindia avec l'aide du CNRS. Le responsable académique en a longtemps été Jean Haritschelhar. C'est aujourd'hui Beñat Oyharçabal qui assure cette fonction. La direction technique est assurée par G. Aurrekoetxea et $X$. Videgain, l'équipe de travail comprenant Kzaskun Etxebeste et Koro Segurola, avec le concours d'AnaMaria Echaide, Jose-Mari Etxebarria et Isaak Atutxa durant les enquêtes, Jose-Antonio Letamendia et Iñaki Camino étant venus renforcer le groupe lors de l'étape du traitement. L'informatisation a compté sur Eđuardo Olea et actuellement Andoni Untzalu. L'unité du CNRS qui aide le projet a été dirigée par Jean Haritschelhar puis Jean-Baptiste Orpustan et Beñat Oyharçabal. 


\section{Bibliographie}

AMADES, J. (1998) : Lorigine des bêtes : petite cosmogonie catalane, Traduction et présentation de Marlène Albert-Llorca, Garae. Hésiode, Paris (l'original catalan date de 1950 : Tradicions Explicatives in Folklore de Catalunya-Rondallistica, Editorial Selecta Barcelona).

AZKUE, R.M. (1935-1947) : Euskalerriaren yakiniza. Literatura popular del Pais Vasco, 4 vol., EspasaCalpe, Madrid.

BLANCHE-BENVENISTE, C. ; JEAN JEAN, C. (1987) : Le français parlé. Transcription \& édition, INALF Didier érudition.

DETIENNE, M. ; VERNANT, J.-P. (1974) : Les ruses de l'intelligence. La métis des Grecs, Flammarion.

HARITSCHELHAR, J. (1969) : Le poète souletin Pierre Topet Etchahun (1786-1682). Contribution à l'étude de la poésie populaire basque du XIX' siècle, Amis du Musée Basque, Bayonne.

HARITSCHELHAR, J. (1970) : L'ceuvre poétique de Pierre Topet-Etchahun, Bilbao.

PETERSON, R.; MOUNFORT, G. et allii. (1984) : Guide des oiseaux d'Europe, Delachaux Niestlé, Neuchâtel-Paris.

PROPP, V (1965): Morphologie du conte, Seuil.

ROLLAND, E. (1877-1911: Faune populaire de France, 13 vol, Paris.

VIDEGAIN, Ch. (1989) : Le vocabulaire de l'élevage en pays d'Oztibarme. Contribution aux archives de l'oralité basque, Thèse de doctorat, Université de Bordeaux III (dir. : J. Haritschelhar, J.-L. Fossat), 2114 p., non publiće. 